\title{
Multi-sensor evaluation of a wooden panel painting using terahertz imaging and shearography
}

\author{
Roger M. Groves ${ }^{* a, b}$, Boris Pradarutti ${ }^{\mathrm{c}}$, Eleni Kouloumpi ${ }^{\mathrm{d}}$, Wolfgang Osten ${ }^{\mathrm{b}}$ and Gunther Notni ${ }^{\mathrm{c}}$ \\ ${ }^{a}$ Faculty of Aerospace Engineering, Delft University of Technology, \\ Kluyverweg 1,2600 GB Delft, The Netherlands; \\ ${ }^{b}$ ITO Institut für Technische Optik, Universität Stuttgart, \\ Pfaffenwaldring 9, 70569 Stuttgart, Germany; \\ ${ }^{c}$ Fraunhofer Institute for Applied Optics and Precision Engineering IOF, \\ Albert-Einstein-Str. 7, 07745 Jena, Germany; \\ ${ }^{\mathrm{d} C}$ Conservation Department, National Gallery - Alexandros Soutzos Museum, \\ 1 Michalacopoulou Str., 11601 Athens, Greece
}

\begin{abstract}
Cultural heritage objects are increasingly being investigated using advanced non-destructive optical measurement techniques. Holographic and speckle interferometry based instrumentation allow dimensional measurement of objects at the tens of nanometer scale. For the structural diagnostics of artwork, double-exposure techniques are often used to locate defects, delaminations, voids and other structural features. Shearography is a speckle interferometry configuration that uses a close-to-common-path shearing interferometer configuration to give a direct sensitivity to displacement gradient at the object surface. This configuration is particularly useful for measurements outside the optical laboratory, as the stability requirements are much reduced compared with holography techniques. Terahertz imaging is a new category of sensor, used to investigate materials using electromagnetic radiation in the 0.1 to $10 \mathrm{THz}$ frequency range. At these frequencies many materials become semi-transparent, so bulk structural diagnostics can be performed. Typically terahertz imaging is performed using a scanning pixel, or multi-pixel, sensor. In this manuscript shearography is first used to identify areas of interest of possible structural anomalies in the artwork. These regions of interest are then studied in more detail using the terahertz imaging instrument. Together the two instruments provide an analysis of both the surface and bulk structural features. The approach is demonstrated experimentally using a wooden panel painting.
\end{abstract}

Keywords: terahertz imaging, shearography, art conservation, structural diagnostics

\section{INTRODUCTION}

Advanced instrumentation is increasingly being applied to measurement problems in cultural heritage. In the field of structural diagnostics of artwork a range of optical techniques are applied to investigating, delaminations, disbonds, voids and other structural features [1]. These measurements guide the continuing conservation of cultural heritage objects, as defect locations can be identified quickly and studied in more detail and deterioration over time can be monitored. Also the response of the cultural heritage object to temperature and humidity changes due to climate change and the museum environment can be monitored.

Shearography is a non-contact field optical measurement technique that measures displacement gradient. To perform a measurement an expanded laser beam is used to illuminate the object, scattered light is optically mixed in a shearing interferometer and the resulting interferogram is recorded by a camera. The recording process is repeated for a second object loading condition and the resulting interferograms are used to calculate the change in displacement gradient. Typical applications of shearography are for defect detection and non-destructive testing for high technology materials and components and for objects with a complex structure.

*r.m.groves@tudelft.nl; phone +31 1527 88230; fax +31 1527 81151; www.tudelft.nl

O3A: Optics for Arts, Architecture, and Archaeology II, edited by Luca Pezzati, Renzo Salimbeni, Proc. of SPIE Vol. 7391, 73910E · ( ) 2009 SPIE · CCC code: 0277-786X/09/\$18 - doi: 10.1117/12.827528 
Terahertz radiation $(0.1-10 \mathrm{THz}, \lambda=3000-30 \mu \mathrm{m})$ is partially absorbed by materials such as paper, canvas and wood and as such is interesting for investigating sub-surface features. In contrast to x-ray radiation it is non-harmful. Terahertz radiation is generated using femto-second laser pulses focused onto a surface emitter, such as p-doped InAs, which is then collimated and focused onto the sample. Coherent detection using an electo-optic crystal is used to determine phase delay or absorption amplitude of the terahertz radiation by the sample.

This manuscript describes a combined shearography-terahertz imaging measurement approach for wooden panel paintings. The entire object is first analysed using shearography and regions of interest are determined from anomalies in the displacement gradient map. These regions are investigated in more detail using slower point-by-point scanning terahertz imaging. Together these techniques provide structural diagnostics of both surface and bulk features.

\section{SHEAROGRAPHY}

Shearography [2] is a full-field non-contact optical measurement technique, closely related to speckle interferometry and holography, that measures surface displacement gradient changes. Because of a high sensitivity, in the tens of $\mu \mathrm{m}$ range, sub-surface defects which produce a strain change at the surface can be detected, in addition to surface defects. Shearography is commonly used in high technology industries, such as aerospace, automotive and precision manufacturing at the design stage, and in aerospace for production quality monitoring and for non-destructive testing at scheduled service intervals.

A typical shearography instrument consists of laser, shearing interferometer and CCD camera. A typical configuration is shown in Figure 1. The object under investigated is illuminated by an expanded laser beam, forming a speckle pattern at the object surface. This speckle pattern is imaged through a shearing interferometer, and the resulting interferogram is recorded by CCD camera. A typical shearing interferometer is a Michelson interferometer with one of the mirrors tilted to produce a double image. Shearography is a double-exposure technique and interferograms recorded before and after object deformation are correlated using computer. Subtraction and intensity rescaling is the simplest technique and this generates correlation fringes showing the deformation gradient. More commonly, phase-shifting techniques [3] and phase unwrapping software [4] are used to obtain the displacement gradient map.

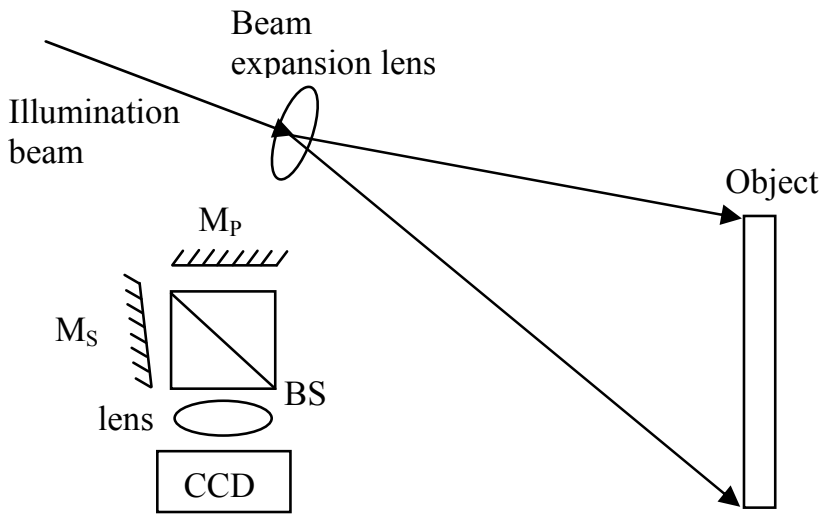

Figure 1 shows a typical shearography experimental layout. The illumination beam is expanded by a lens to illuminate the surface of the object. Light scattered by the object is optically mixed in the interferometer and the resulting interferogram is imaged onto the camera chip, $C C D$, by a lens. The interferometer components are beamsplitter, $B S$, phase shifting mirror, $M_{P}$, and shearing mirror, $M_{S}$.

Shearography is sensitive to a component of displacement gradient determined by the illumination and viewing directions and by the direction of applied shear. Direction and magnitude adjustments of the shearing interferometer control the displacement gradient component measured and the measurement sensitivity. The most common configuration is sensitive to one of the out-of-plane displacement gradients, as given by Equation 1: 


$$
\Delta \phi_{(x, y)}=\frac{4 \pi}{\lambda} \frac{\delta w}{\delta x}(x, y) \text {, or } \Delta \phi_{(x, y)}=\frac{4 \pi}{\lambda} \frac{\delta w}{\delta y_{(x, y)}} d y
$$

where $\Delta \varphi$ is the phase change in the interferometer, $\lambda$ is the optical wavelength, $\delta w / \delta x$ is the out-of-plane displacement differentiated in the horizontal direction, $\delta w / \delta y$ is the out-of-plane displacement differentiated in the vertical direction, and $d x$ and $d y$ are the horizontal and vertical shear magnitudes, respectively.

\section{TERAHERTZ IMAGING}

One technique for generating short terahertz pulses is by focusing femto-second laser pulses onto a terahertz surface emitter [5], as shown in Figure 2. Femto-second pulses are separated into a pump beam and a gate beam. The laser pulses pass through a chopper, to synchronise phase-sensitive timing, and a half-wave plate, rotated to maximise absorption and hence terahertz emission at the subsequent terahertz surface emitter, e.g. a p-doped InAs. The terahertz pulses are collimated by an off-axis parabolic mirror and focused onto the sample by a lens. On the other side of the sample a second lens and parabolic mirror focuses the terahertz pulses onto an electro-optic crystal.

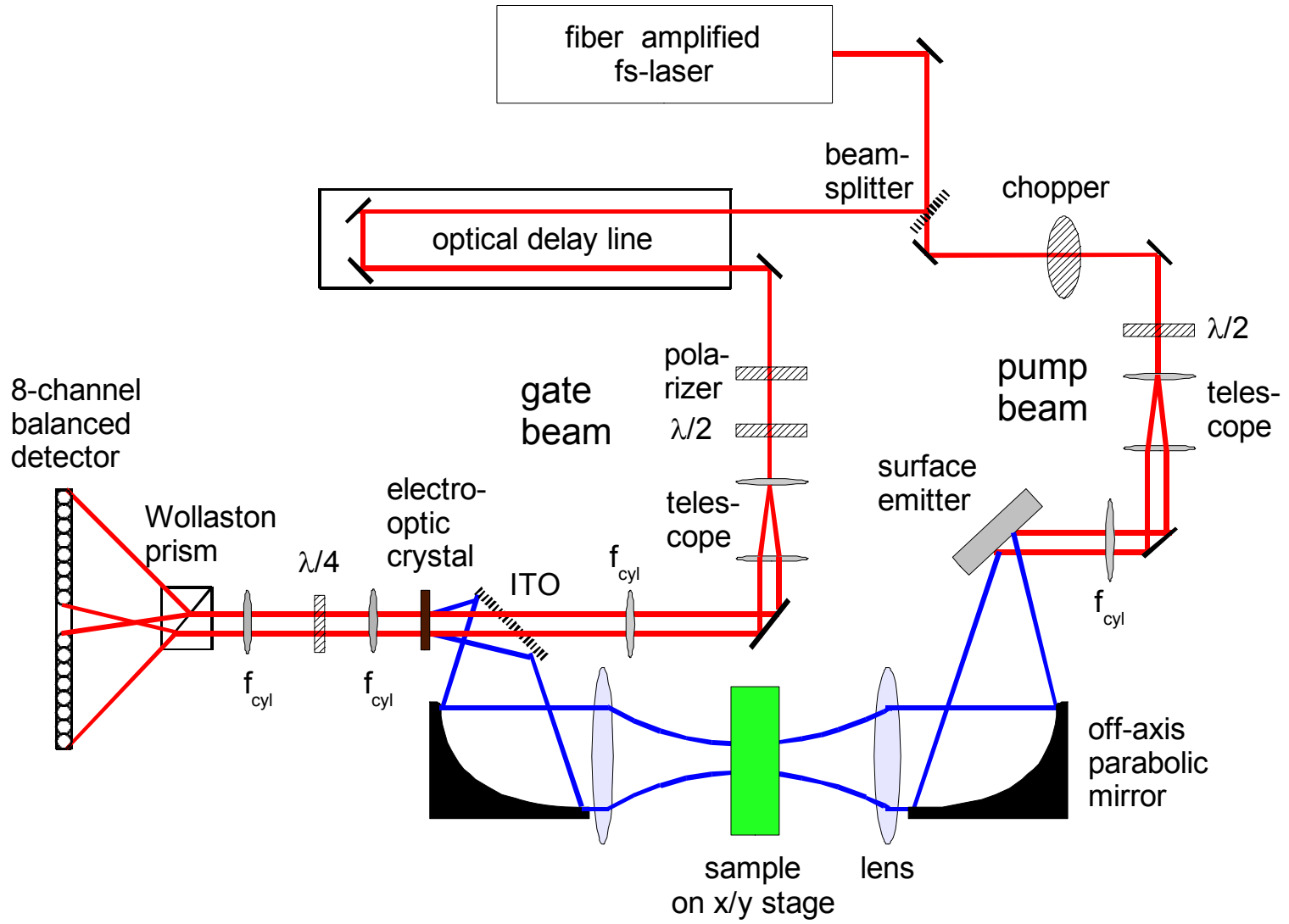

Figure 2 shows the 8-channel THz imaging system based on a fibre amplified fs-laser: The fs pulses are split into a pump and a gate beam. The pump beam excites ultra-short THz-pulses, which are focussed inside the sample. The transmitted pulses are imaged into the detection crystal. The gate beam probes the THz electric field inside the electro-optic crystal by the Pockel's effect. The resulting change in polarization is converted in an intensity change by a quarter-wave plate and a Wollaston prism and is detected by a balanced detector.

The linearly polarised gate beam is first directed along an optical delay line before being focused onto the same electrooptic crystal. Additionally, a half-wave plate is used to align the polarisation of the gate beam before the crystal. The terahertz radiation is detected by the linear electro-optic effect. The terahertz pulse induces a rotation in the polarisation of the gate beam, which can be converted to an intensity change by a quarter-wave plate and polarisation dependent beamsplitter (e.g. a Wollaston prism). This intensity change can be measured by a balanced detector without laser power noise. Temporally the gate pulse is much shorter than the terahertz pulse, so the detector configuration samples part of 
the terahertz pulse. Adjusting the optical delay line allows the time-resolved sampling of the terahertz pulses. A more detailed description of the time-resolved sampling of ultra-short terahertz pulses is given in $[6,7]$.

Single channel detection, with the object translated in the focal plane by an $x / y$ stage, is commonly used to perform terahertz imaging. Multiple channel detection, with a limited number of channels, is also possible, e.g. [8].

\section{INSTRUMENTATION}

The shearography instrumentation was designed for measurements on artwork as part of the EU FP6 Project MultiEncode (006427) and follows the experimental configuration shown in Figure 1. The object is illuminated by the expanded beam from a Coherent Verdi-V10 laser $(532 \mathrm{~nm}$, optical power adjustable from $\sim 50 \mathrm{~mW}$ to $10 \mathrm{~W})$. A typical optical power used for the artwork samples described in this paper would be $500 \mathrm{~mW}$. The optical head consists of a shearing Michelson interferometer, camera lens (25 mm focal length) and a Basler A102f CCD camera (1392 x 1040 pixels, pixel size $6.45 \times 6.45 \mu \mathrm{m}^{2}$, Firewire interface). The shearing interferometer is composed of a broadband beamsplitter cube, shearing mirror, which can be tilted horizontally or vertically, and a phase-shifting mirror which is controlled by a Linos PSP-1 piezo-electric element. The optical head is interfaced to the PC via an electronics control box which contains the PZT amplifier, Firewire power supply and a USB DAC card (National instruments NI-6008). One Firewire cable and one USB cable connect directly to the PC. The instrument is operated using software developed using Labview software (National Instruments) and controls instrument setting, phase-shifting and image capture and performs image processing and live display of phase maps and data archiving. To avoid difficulties with image noise when measuring samples with difficult surface reflectivities, Extended Dynamic Range (EDR) imaging for shearography is implemented [9]. EDR imaging for shearography was specifically developed for measuring wooden panel paintings with gold and dark earth pigments, so that phase maps with acceptable noise could be obtained. Once the measurement procedure is started the software records reference interferograms, applies a controlled loading to the painting using infra-red lamps and before recording further interferograms. Wrapped and unwrapped phase maps are displayed in realtime and the phase maps are archived to the hard disk. A typical measurement time is $300 \mathrm{~s}$, with interferograms recorded typically every $10 \mathrm{~s}$. Figure 3 shows the experimental configuration and the software user interface.

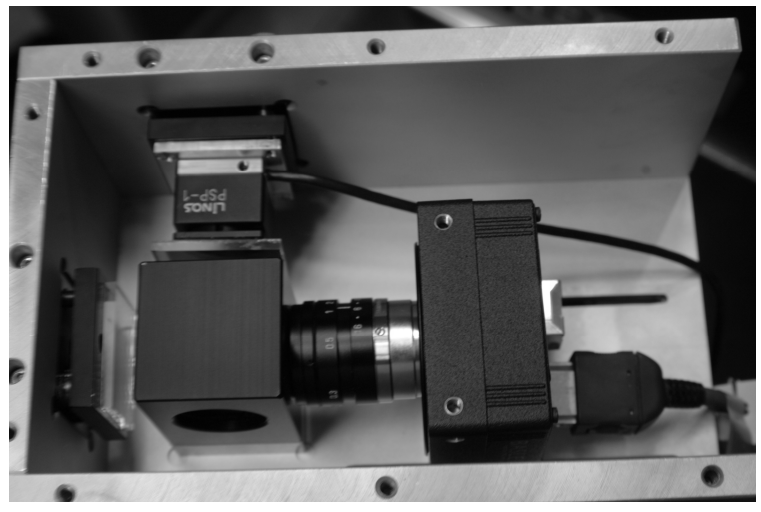

(a)

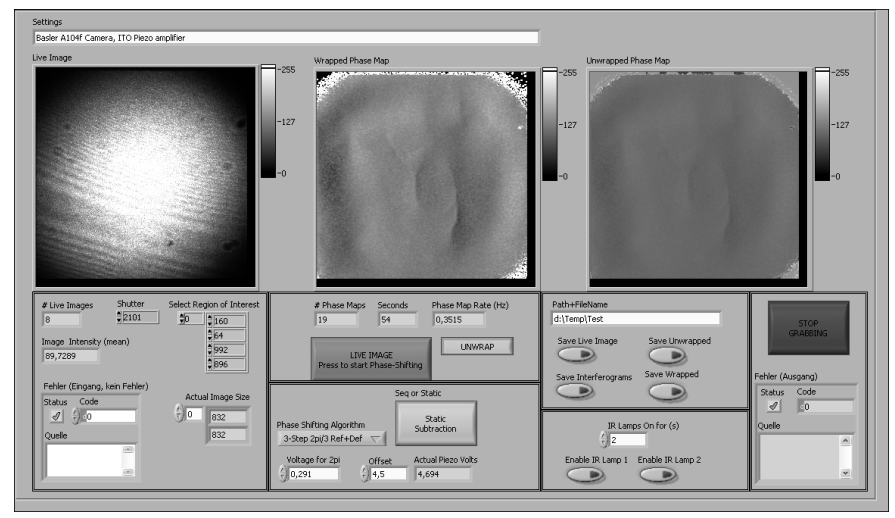

(b)

Figure 3(a) shows the interferometer head composed of shearing Michelson interferometer, lens and camera and (b) the user interface for the software showing from left to right live display, wrapped phase map and unwrapped phase map.

The ultra-short pulse terahertz imaging system follows the schematic in Figure 2. The system is based on a fibreamplified femtosecond laser system [10] delivering up to $10 \mathrm{~W}$ at $1060 \mathrm{~nm}$. The pulse length is about $100 \mathrm{fs}$ and the pulse repetition rate is $75 \mathrm{MHz}$. Approximately $96 \%$ of the energy is directed to the pump beam with the remaining $4 \%$ directed to the gate beam. Both beams are expanded by a telescope and focused by cylindrical lenses to achieve line foci. The ultra-short pulses emitted by the surface emitter (p-doped InAs) are used to illuminate the sample, with a demagnification of 0.9. The terahertz pulses transmitted through the sample are imaged onto the electro-optic crystal (CdTe, $1 \mathrm{~mm}$ thickness, [11]) for terahertz detection. Overall the THz-optics gives a magnification equal to one [12]. The gate beam is focused as a line onto the detection crystal to probe the electric field of the terahertz pulse by the Pockel's effect position dependently. The change in polarization in the gate beam, induced by the terahertz pulse, probed position-dependently using a quarter-wave plate, Wollaston prism and 8-channel balanced detector. 


\section{RESULTS}

A wooden panel painting with realistic construction was developed to test the performance of structural diagnostics measurement techniques. The protocol for construction was based on the traditional techniques described by famous art masters of the period, such as Dionysios ek Fourna and Cennino Cennini [13]. The painting is composed of a number of layers upon a wooden base containing a knot with a crack. Starting from the substrate, the principle layers added were (i) preparation layer (gesso and animal glue), (ii) bole, (iii) layer of gold, (iv) paint layer (pigment and egg yolk) and (v) varnish. The sample is 198-201 mm wide, 101-112 mm high and 13-14 mm thick, including a paint layer with thickness of $0.5-1 \mathrm{~mm}$. Note these dimension ranges are due to irregularities in the shape of the sample. The sample surface deviated by $4 \mathrm{~mm}$ from a flat surface at its maximum deviation.

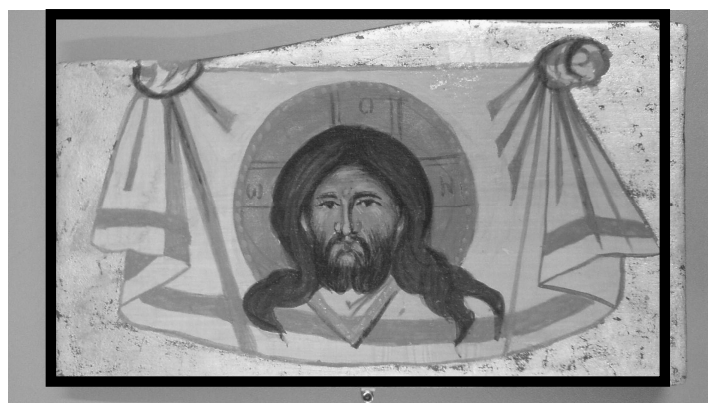

(a)

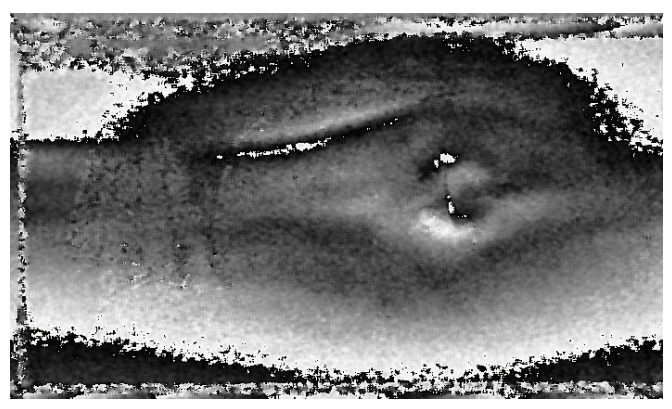

(b)

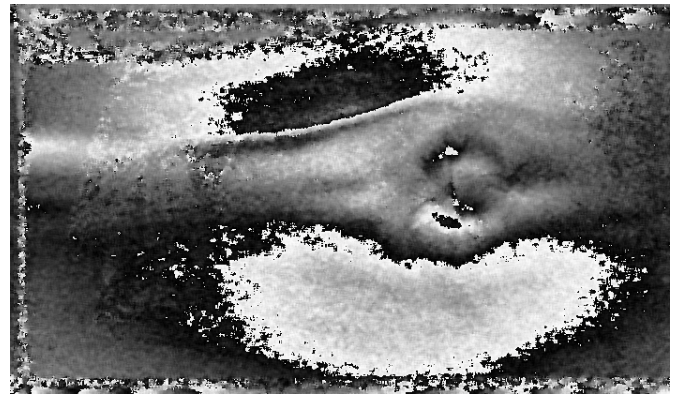

(d)

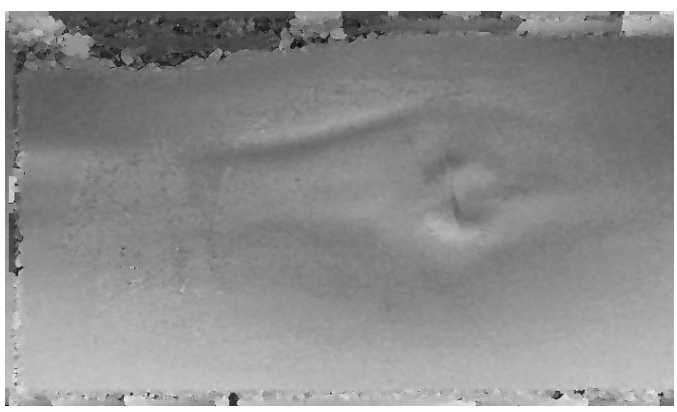

(c)

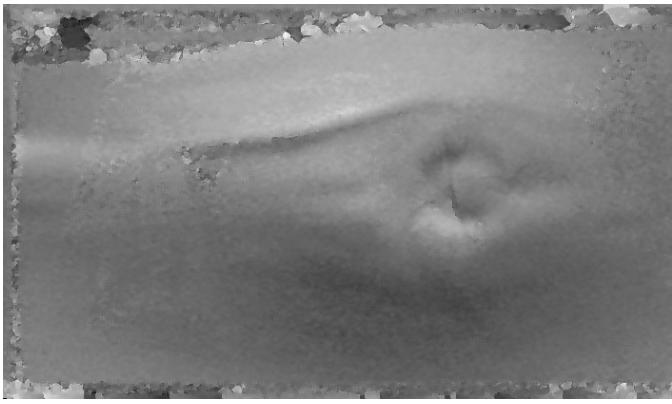

(e)

Figure 4 shows (a) a photograph of the painting showing the field of view for shearography. (b) is a wrapped phase map and (c) is an unwrapped phase map, after thermal loading from front and back. (e) and (f) are respectively wrapped and unwrapped phase maps, recorded after thermal loading from the front. In all cases the images were recorded using the shearography instrument, $183 \mathrm{~s}$ after thermal loading.

The wooden panel painting was analysed using the shearography instrument described in Section 4. The object was placed $1 \mathrm{~m}$ from the sensor and was illuminated using a $300 \mathrm{~mW}$ expanded laser beam. The shear applied in the interferometer was $5 \mathrm{~mm}$, perpendicular to the wood grain. The object was thermally loaded using two $150 \mathrm{~W}$ infra-red lamps and heating the sample from front and back for 5 seconds. The measurement time was 300 seconds. Wrapped and 
unwrapped phase maps from the instrument are shown in Figure 4. The results clearly show the position of the knot and the strain field due to the wood grain. The shearography sensor measures the sample with a vertical resolution of $5 \mathrm{~mm}$ and a horizontal resolution of $0.6 \mathrm{~mm}$.

The terahertz measurements were performed using the terahertz imaging sensor described in Section 4 . The sample was placed on a motorized $x / y$ translation stage and a region of interest, selected from the shearography data, was moved through 51 (horizontal) x 150 (vertical) positions at the terahertz line focus. At each position the optical delay line was scanned to perform simultaneous 8-channel detection. The resolution obtained was 408 (horizontal) by 150 (vertical) pixels in the electric field traces for both time-delay and pulse amplitude. This is equivalent to a resolution of $0.3 \times 0.5$ $\mathrm{mm}^{2}$. The results are shown in Figure 5 .

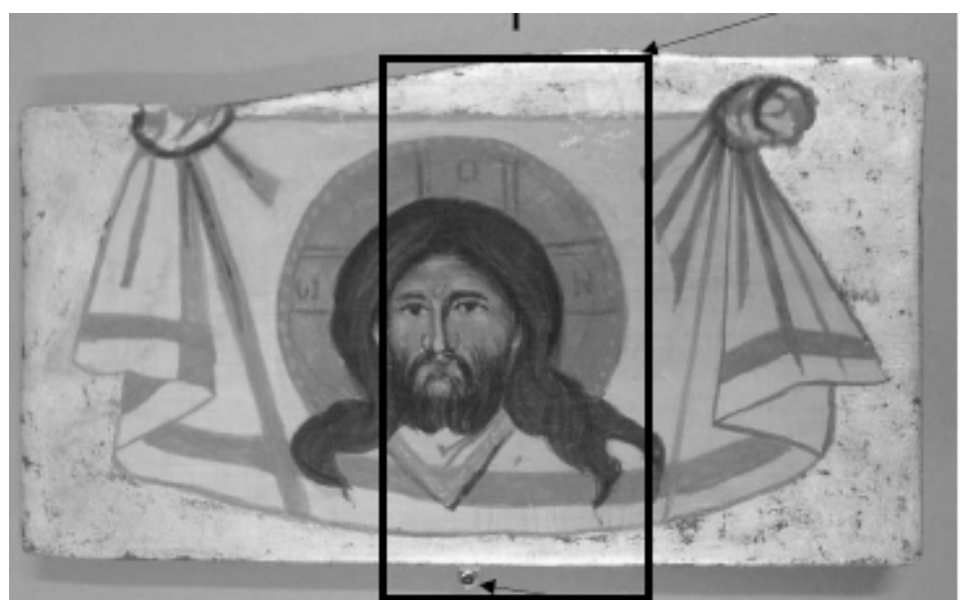

(a)

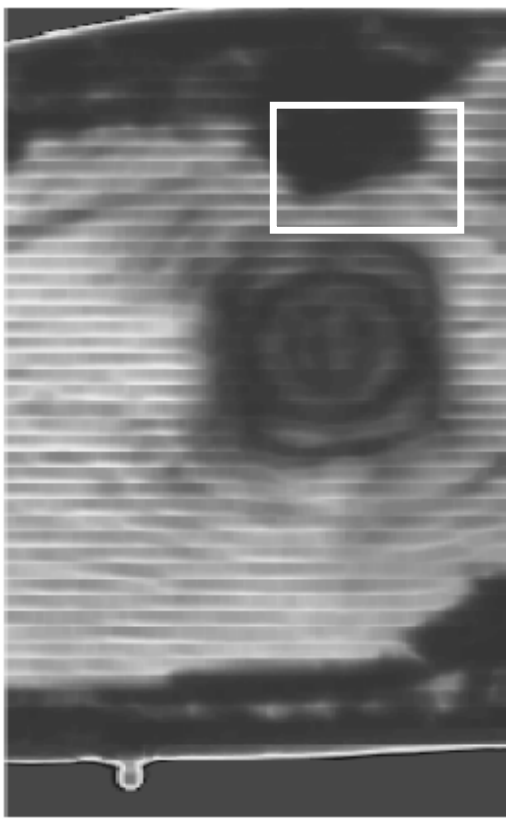

(b)

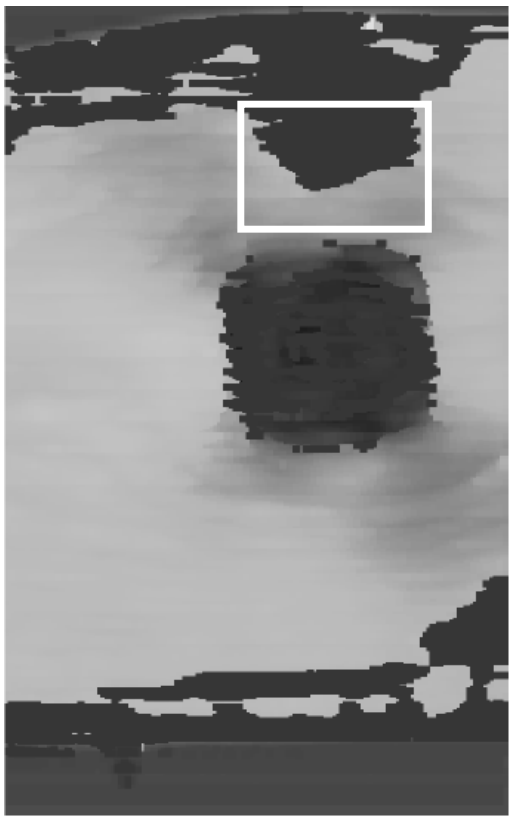

(c)

Figure 5 shows (a) a photograph of the painting, indicating the field of view of the terahertz sensor. (b) is the amplitude analysis and (c) is the time-delay phase analysis. The white rectangle indicates a possible defect region, undetected by shearography. 


\section{DISCUSSION}

The shearography results show the wooden knot, wood grain and the crack can be reliably detected using both the front and front/back thermal loading. The front thermal loading configuration would be particularly useful when the artwork is fixed to a wall, as measurements could be made in-situ. The measurement speed is quite fast for shearography with defect maps obtained in real time through the object cooling cycle once the instrument set-up procedure is completed. The sample measured for this paper showed an interesting region of interest for further investigation with terahertz imaging around the knot.

Terahertz imaging provides information through the bulk of the sample. Amplitude analysis shows the pixel by pixel absorption of terahertz radiation by the sample, confirming the presence of the long defect to the above right of the head, the knot and some of the smaller defects due to the wood grain. The time-delay analysis shows the wood knot strongly. Both terahertz analysis techniques identify a region above the knot with has a distinct signature. The defect is not detectable by shearography or by visual inspection of the painting. This type of region would be highlighted to the conservator for further detailed investigation. Very sensitive objects can be investigated using terahertz radiation which does not cause localized heating or damage, due to the low energy of the radiation. Unlike x-ray techniques terahertz radiation is safe for the instrument user.

The results show that an integrated shearography-terahertz imaging approach could be used for the analysis of cultural heritage objects. A first analysis of the complete artwork is performed using shearography. Typically, for a portable instrument, objects with a size of up to $300 \times 300 \mathrm{~mm}^{2}$ can be measured using a Class IIIb Nd:YAG laser $(<500 \mathrm{~mW})$. For larger artworks, measurements can be made of sub-regions with a size of up to $300 \times 300 \mathrm{~mm}^{2}$. Shearography can be used reliably outside the optics laboratory with the sensor mounted a tripod, so measurements in museums and churches are possible. Depending on the shearography results obtained, a more detailed analysis of movable cultural heritage objects can be performed in the optics laboratory using the terahertz imaging technique.

\section{CONCLUSIONS}

The manuscript demonstrates the successful implementation of a combined shearography-terahertz imaging measurement approach for the structural diagnostics of wooden panel paintings. The shearography sensor provides the surface and sub-surface information about the artwork and the terahertz imaging sensor provides information on the bulk of the sample.

\section{ACKNOWLEDGEMENTS}

The construction of the shearography sensor was supported by the European Union funded MULTI-ENCODE (Multifunctional Encoding System for the Assessment of Movable Cultural Heritage) FP6 Project (006427 SSPI). The construction of the THz imaging system was supported by the FhG internal programs under grant no. MAVO 813907.

\section{REFERENCES}

[1] Fotakis, C., Anglos, D., Zafiropulos, V., Georgiou, S. and Tornari, V., [Lasers in the preservation of cultural heritage], CRC Press, Boca Raton (2006).

[2] Steinchen, W., and Yang, L., [Digital shearography], SPIE Press, Bellingham (2001).

[3] Creath, K., "Temporal phase measurement methods", Robinson, D. W., and Reid, G. T., editors, in: Interferogram analysis, digital fringe measurement methods, Institute of Physics, Bristol, pp. 94-140 (1993)

[4] Ghiglia, D. C., and Pritt, M. D., [Two-Dimensional Phase Unwrapping], J. Wiley, New York (1998)

[5] Sakai, K., Ed., [Terahertz Optoelectronics], Springer Berlin Heidelberg (2005)

[6] Bakker, H. J., Cho, G., Kurz, H., Wu, Q. and Zhang, X, "Distortion of terahertz pulses in electro-optic sampling", J. Opt. Soc. Am. B 15, 1795-1801, (1998)

[7] Gallot, G. and Grischkowsky, D., "Electro-optic detection of terahertz radiation", J. Opt. Soc. Am. B 16, 1204$1212(1999)$ 
[8] Pradarutti, B., Müller, R., Matthäus, G., Brückner, C., Riehemann, S., Notni, G., Nolte, S. and Tünnermann, A., "Multichannel balanced electro-optic detection for Terahertz imaging", Opt. Exp. 15, 17652-17660 (2007)

[9] Groves, R. M,, Pedrini, G. and Osten, W., "Real-Time Extended Dynamic Range Imaging in Shearography", Appl. Opt. 47, 5550-5556 (2008)

[10] Matthäus, G., Schreiber, T., Limpert, J., Nolte, S., Torosyan, G., Beigang, R., Riehemann, S., Notni, G. and Tünnermann, A., "Surface-emitted THz generation using a compact ultrashort pulse fiber amplifier at 1060 nm", Opt. Comm. 261, 114-117 (2006)

[11] Pradarutti, B., Matthaus, G., Riehemann, S., Notni, G., Nolte, S. and Tünnermann, A, "Highly efficient terahertz electro-optic sampling by material optimization at $1060 \mathrm{nm",} \mathrm{Opt.} \mathrm{Comm.} \mathrm{281,} \mathrm{5031-5035} \mathrm{(2008).}$

[12] Brückner, C., Pradarutti, B., Müller, R., Notni, G. and Tünnermann, A., "Design and Evaluation of a THz Time Domain Imaging System using Standard Optical Design Software", Appl. Opt. 47, 4994-5006 (2008)

[13] Kouloumpi, E., Moutsatsou, A. P., Trompeta, M., Olafsdottir, J., Tsaroucha, C., Terlixi, A. V., Groves, R. M., Georges, M., Hustinx, G.-M. and Tornari, V., "Laser-based structural diagnosis: A museum's point of view", in: 7th International Conference on Lasers in the Conservation of Artworks, Taylor \& Francis, Madrid, (2008) 